\title{
Évaluation de l'innocuité des vaccins dans le Programme universel de vaccination contre la grippe de l'Ontario, de 2012-2013 à 2014-2015
}

\author{
Harris $\mathrm{T}^{1 *}$, Wong K ${ }^{1}$, Nair J11, FediurekJ ${ }^{1}$, Deeks $\mathrm{SL}^{1,2}$
}

\section{Résumé}

Contexte : Le vaccin antigrippal est recommandé pour prévenir la morbidité et la mortalité liées à la grippe. Après la commercialisation, la surveillance des effets secondaires suivant l'administration du vaccin antigrippal est essentielle pour surveiller l'innocuité des vaccins, orienter la planification et l'évaluation du programme d'immunisation, et renforcer la confiance à l'égard de l'immunisation.

Objectif : Présenter un résumé des effets secondaires suivant l'immunisation (ESSI) signalés après l'administration de vaccins antigrippaux administrés dans le cadre du Programme universel de vaccination contre la grippe de l'Ontario.

\section{Méthodologie : Les effets secondaires suivant l'immunisation qui surviennent après} I'administration de vaccins antigrippaux entre le $1^{\text {er }}$ septembre 2012 et le 31 août 2015 ont été extraits du Système d'information sur la santé publique intégré (SISP-i) le 1 1er septembre 2015. Les effets ont été regroupés en fonction des définitions de surveillance provinciales. Les taux de déclaration ont été calculés en prenant comme dénominateur les estimations démographiques des provinces ou le nombre net de doses distribuées. La définition standard des effets secondaires graves suivant l'immunisation de l'Organisation mondiale de la Santé a été utilisée.

Résultats : II a été distribué 12,1 millions de doses de vaccin antigrippal en Ontario et 528 effets secondaires suivant l'immunisation ont été signalés après l'administration des vaccins antigrippaux au cours des trois saisons. Les taux de déclaration annualisés étaient de 4,4 cas pour 100000 doses distribuées, avec une forte tendance à la baisse au fil du temps $(p<0,05)$. L'âge médian était de 39,6 ans (plage de 6 mois à 96 ans); les enfants de moins de quatre ans avaient le plus haut taux de déclaration (3,5 pour 100000 habitants). Une déclaration disproportionnée chez les femmes a été observée $(76,5 \%)$, plus particulièrement chez les femmes âgées de 18 ans et plus. Les effets les plus souvent signalés étaient des réactions au site d'injection (36,2\% des déclarations). D'autres effets étaient des réactions allergiques cutanées (21,1\%) et des éruptions cutanées (17,3\%). Les effets secondaires suivant l'immunisation graves étaient rares, avec un taux de déclaration de 1,6 cas pour un million de doses distribuées.

Conclusion : Cette évaluation a révélé un faible taux d'effets secondaires déclarés après l'administration de vaccins antigrippaux en Ontario. La plupart des effets signalés étaient légers et se sont complètement dissipés. Les résultats ont été conformes au très bon profil d'innocuité des vaccins antigrippaux.

\author{
Affiliations \\ 1 Santé publique Ontario, Toronto \\ (Ontario) \\ ${ }^{2}$ Dalla Lana School of Public \\ Health, Université de Toronto, \\ Toronto (Ontario)
}

*Correspondance : tara.harris@ oahpp.ca

Citation proposée : Harris T, Wong K, Nair J, Fediurek J, Deeks SL. Évaluation de l'innocuité des vaccins dans le Programme universel de vaccination contre la grippe de l'Ontario, de 2012-2013 à 2014-2015. Relevé des maladies transmissibles au Canada 2016;42:202-8. https://doi.org/10.14745/ccdr.v42i09a04f

\section{Introduction}

La grippe est une infection respiratoire qui cause environ 12200 hospitalisations et 3500 décès chaque année au Canada (1). La vaccination annuelle contre la grippe saisonnière est le moyen le plus efficace de prévenir la grippe et ses complications. II y a plusieurs vaccins antigrippaux homologués au Canada pour

les personnes de six mois et plus. Ces produits comprennent les vaccins trivalents et quadrivalents inactivés (y compris les préparations contenant un adjuvant et les préparations à dosage élevé) et les vaccins trivalents et quadrivalents vivants atténués avec diverses indications selon l'âge et le statut immunitaire $(1,2)$. Le Comité consultatif national de 
l'immunisation (CCNI) recommande le vaccin antigrippal pour toutes les personnes de six mois et plus qui ne présentent pas de contre-indications, et plus particulièrement les personnes présentant un risque élevé de complications ou d'hospitalisation liées à la grippe et les personnes susceptibles de transmettre la grippe à des sujets à risque élevé (1). L'Ontario a un Programme universel de vaccination contre la grippe financé par l'État depuis 2000 (3). Tous les Ontariens âgés de six mois et plus, qui vivent, travaillent ou se rendent à l'école dans la province sont admissibles au vaccin antigrippal annuel financé par l'État. La plupart des vaccins antigrippaux dans la province sont administrés dans le cadre du Programme universel de vaccination contre la grippe. Cependant, les produits de vaccination dont l'utilisation est autorisée, mais qui ne sont pas compris dans le programme public peuvent être achetés à titre privé.

Les vaccins antigrippaux sont en général sûrs et bien tolérés. Dans le cas des vaccins antigrippaux administrés par injection intramusculaire, l'effet secondaire le plus courant est la douleur au site d'injection, qui touche entre $40 \%$ à $60 \%$ des adultes en bonne santé, mais elle est généralement bénigne et disparaît en quelques jours $(4,5)$. Une plus grande fréquence de réactions au point d'injection est observée pour les préparations avec adjuvant et à dosage élevé. Les effets secondaires graves sont rares et comprennent l'anaphylaxie et le syndrome de Guillain-Barré (1). La surveillance après commercialisation des vaccins antigrippaux est essentielle pour continuer à démontrer I'innocuité des vaccins au fil du temps, pour orienter l'évaluation et pour renforcer la confiance à l'égard des programmes d'immunisation contre la grippe. Les renseignements découlant de la surveillance de la santé publique des effets secondaires suivant l'immunisation fournissent des renseignements pertinents et opportuns permettant d'aborder les préoccupations concernant l'innocuité des vaccins, qui se sont avérées être I'un des principaux obstacles ayant une influence sur le taux d'acceptation des vaccins dans la population générale (6-8) et les travailleurs de la santé (9-11).

Notre objectif est de résumer les effets secondaires suivant I'immunisation contre la grippe signalés en Ontario au cours des trois dernières saisons grippales afin d'appuyer une évaluation globale du Programme universel de vaccination contre la grippe de l'Ontario.

\section{Méthodologie}

\section{Définitions}

Un effet secondaire suivant l'immunisation confirmé est un effet signalé chez un sujet vacciné après une immunisation qui ne peut pas être clairement attribué à d'autres causes. Une relation de cause à effet avec l'administration du vaccin n'a pas besoin d'être prouvée (12). Les effets secondaires sont définis selon des critères de surveillance provinciales propres à un effet (12). Les effets secondaires suivant l'immunisation sont définis comme étant graves s'ils répondent à la définition de l'Organisation mondiale de la Santé, qui précise qu'un effet secondaire suivant I'immunisation grave est un effet qui entraîne la mort, met la vie en danger, exige l'hospitalisation du patient ou la prolongation de son hospitalisation, entraîne une invalidité ou une incapacité persistante ou marquée, ou entraîne une déficience ou malformation congénitale $(13,14)$. D'autres événements médicaux importants comprennent l'anaphylaxie, l'encéphalite, l'encéphalomyélite aiguë disséminée, la myélite, la méningite, le syndrome de Guillain-Barré, l'ataxie cérébelleuse aiguë et la thrombocytopénie. Ces événements ne répondent pas à la définition susmentionnée d'effets graves, et ont été évalués et présentés séparément. Les déclarations d'effets pris en charge comme anaphylaxie ont été évaluées au moyen de la définition de cas de Brighton Collaboration et des niveaux de certitude des diagnostics (15).

En Ontario, la déclaration d'effets secondaires suivant l'immunisation par des professionnels de la santé particuliers (p. ex. les médecins, les infirmières et les pharmaciens) est prescrite par les lois provinciales sur la santé publique (16); toutefois, une déclaration volontaire de sujets vaccinés ou de leurs personnes soignantes se fait également. Les déclarations d'effets secondaires suivant l'immunisation sont reçues par les bureaux de santé publique locaux, qui examinent les déclarations et saisissent des renseignements, conformément aux lignes directrices en matière de surveillance provinciale dans le Système d'information sur la santé publique intégré (SISP-i), le système de déclaration électronique des maladies à déclaration obligatoire et des effets secondaires suivant l'immunisation en Ontario.

L'examen portait sur tous les effets secondaires suivant l'immunisation déclarés après l'administration de vaccins antigrippaux dans le cadre du Programme universel de vaccination contre la grippe entre le $1^{\text {er }}$ septembre 2012 et le 31 août 2015. Les données ont été extraites du SISP-i le $1^{\mathrm{er}}$ septembre 2015. Les produits de vaccination utilisés dans le cadre du Programme universel de vaccination contre la grippe entre 2012-2013 et 2014-2015 étaient des vaccins trivalents inactivés, dont : Fluviral ${ }^{\circledR}$, Agriflu $^{\circledR}$, Vaxigrip ${ }^{\circledR}$, Fluzone ${ }^{\circledR}$ (2014-2015 seulement) et Fluad ${ }^{\circledR}$ (pour les personnes de 65 ans et plus qui résident dans des établissements de soins de longue durée). Les déclarations suivant l'administration d'un vaccin antigrippal vivant atténué ont été exclues, puisqu'elles ne représentaient qu'un très petit nombre des déclarations $(n=4)$ et ne faisaient pas partie du Programme universel de vaccination contre la grippe au cours de la période de déclaration.

Les proportions ont été basées sur les déclarations ayant des données complètes dans le SISP-i. Par conséquent, le dénominateur varie selon la variable. Les tendances temporelles ont été évaluées par saison grippale, définie comme étant du $1^{\text {er }}$ septembre au 31 août aux fins de la surveillance provinciale des effets secondaires suivant l'immunisation contre la grippe. Les taux de déclaration des effets secondaires suivant l'immunisation ont été calculés en se servant de dénominateurs basés à la fois sur le nombre de doses distribuées et sur la population. Les taux de déclaration ont été calculés au fil du temps et par type d'effet en utilisant comme dénominateur le nombre net de doses distribuées dans le cadre du programme financé par l'État. Ce nombre a été calculé en fonction des données sur les estimations du nombre net de vaccins distribués fournies par le Service d'approvisionnement médico-pharmaceutique du gouvernement de l'Ontario et ajustées pour tenir compte des vaccins gaspillés et des vaccins réutilisables retournés. Les taux de déclaration ont été calculés par groupe démographique (p. ex. l'âge, le sexe et la géographie) en se servant de dénominateurs basés sur la 
population en l'absence de renseignements sur la répartition des doses au sein de ces groupes. Les dénominateurs de la population étaient fondés sur les estimations de 2012 et de 2013 pour les saisons grippales 2012-2013 et 2013-2014, respectivement, et sur les projections de 2014 pour la saison 2014-2015 $(17,18)$. Une analyse statistique a été effectuée à I'aide de la version 9.3 du système SAS et de Microsoft Excel 2010. Les tendances relatives aux taux d'incidence pendant la période entière de l'étude ont été évaluées selon la régression de Poisson, et les valeurs de $p$ inférieures à 0,05 ont été considérées comme statistiquement significatives. Ce projet a été approuvé par le Comité d'éthique de Santé publique Ontario.

\section{Résultats}

Un nombre de 528 effets secondaires suivant l'immunisation ont été déclarés après l'administration de vaccins antigrippaux dans le cadre du Programme universel de vaccination contre la grippe de l'Ontario entre le $1^{\text {er }}$ septembre 2012 et le 31 août 2015. Au cours de cette période, plus de 12,1 millions nets de doses de vaccins du Programme universel de vaccination contre la grippe ont été distribués, pour un taux de déclaration annualisé de 4,4 cas pour 100000 doses distribuées. Une forte tendance à la baisse a été observée dans le taux de déclaration par saison, avec une baisse marquée du taux de déclaration en 2014-2015 par rapport à 2012-2013 et à 2013-2014 ( $p<0,05)$ (figure 1).

Figure 1 : Nombre d'effets secondaires suivant l'immunisation contre la grippe et taux de déclaration (pour 100000 doses distribuées) en Ontario, de 2012-2013 à 2014-2015

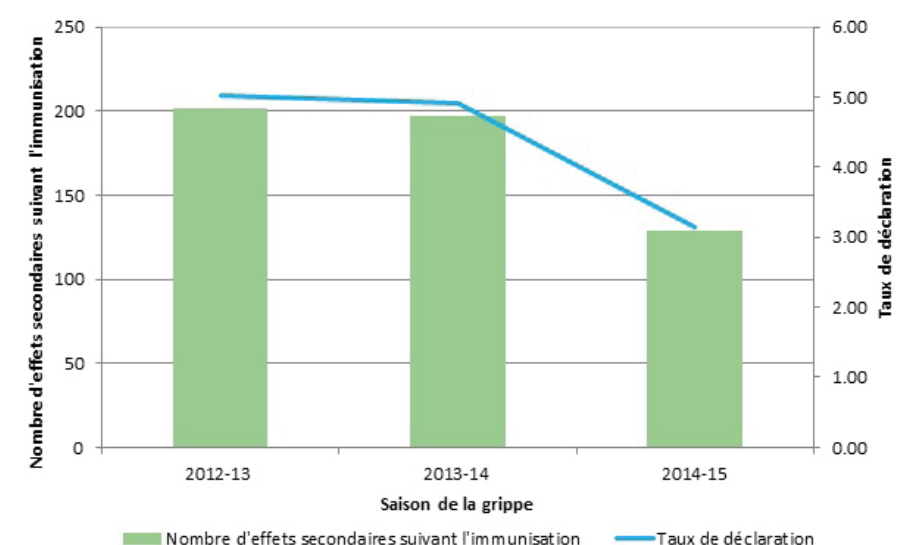

Remarques :

1. Le nombre de déclarations d'effets secondaires suivant l'immunisation comprend uniquement les déclarations effectuées après les vaccins antigrippaux financés par l'État (il exclut par exemple les effets secondaires suivant l'immunisation après un vaccin antigrippal vivant atténué) et est exact en date du 1er septembre 2015

2. Le taux de déclaration est calculé à l'aide du dénominateur suivant : doses de vaccin antigrippal financé par l'État distribuées par le Service d'approvisionnement médico-pharmaceutique du gouvernement de l'Ontario entre le $1^{\text {er }}$ septembre 2012 et le 31 août 2015

Les personnes ayant des effets secondaires suivant I'immunisation avaient de 6 mois à 96 ans (âge médian : 39,6 ans). Plus de la moitié de toutes les déclarations $(58,2$

$\%)$ ont été observées chez les adultes âgés de 18 à 64 ans. Cependant, les taux de déclaration les plus élevés en fonction de l'âge ont été observés chez les enfants de moins de 4 ans et ceux de 5 à 9 ans (3,5 et 2,8 pour 100000 habitants, respectivement) (tableau 1). Les taux de déclaration par groupe d'âge ont diminué au cours des trois saisons, avec une baisse significative chez les enfants de moins de 4 ans et les adultes de 50 à 64 ans. Une déclaration disproportionnée a été observée chez les femmes (76,5\% dans l'ensemble), en particulier dans les tranches d'âge chez les adultes (93,3\% et 86,5\% chez les 18 à 49 ans et chez les 50 à 64 ans, respectivement). Le rapport femmes-hommes le plus élevé du taux de déclaration a été observé chez les adultes âgés de 18 à 49 ans et de 50 à 64 ans (13,8 \% et $6,2 \%$, respectivement). Sur le plan géographique, les taux de déclaration par bureaux de santé publique variaient largement, allant de 0 à 9,9 pour 100000 habitants, sans tendance observée.

\section{Tableau 1 : Nombre d'effets secondaires suivant I'immunisation contre la grippe, pourcentage et taux de déclaration en Ontario, par groupe d'âge, de 2012-2013 à 2014-2015}

\begin{tabular}{|r|r|r|r|r|}
\hline $\begin{array}{c}\text { Catégorie } \\
\text { d'âge } \\
\text { (années) }\end{array}$ & Nombre $^{2}$ & $\begin{array}{c}\text { Pourcentage } \\
\text { d'effets } \\
\text { secondaires } \\
\text { suivant } \\
\text { I'immunisation }\end{array}$ & $\begin{array}{c}\text { Pourcentage } \\
\text { de } \\
\text { population }\end{array}$ & $\begin{array}{c}\text { Taux de } \\
\text { déclaration } \\
\text { (pour } \\
100 \text { 000 } \\
\text { habitants) }\end{array}$ \\
\hline$\leq 4$ & 75 & 14,3 & 5,3 & 3,49 \\
\hline 5 à 9 & 61 & 11,6 & 5,4 & 2,78 \\
\hline 10 à 17 & 22 & 4,2 & 9,0 & 0,59 \\
\hline 18 à 49 & 180 & 34,2 & 43,8 & 1,00 \\
\hline 50 à 64 & 126 & 24,0 & 20,9 & 1,50 \\
\hline 65 à 79 & 50 & 9,5 & 11,5 & 1,11 \\
\hline 80 et plus & 12 & 2,3 & 4,2 & 0,72 \\
\hline Total & 526 & 100,0 & 100,0 & 1,29 \\
\hline
\end{tabular}

${ }^{1}$ Âge : Date de l'administration du vaccin antigrippal - date de naissance

${ }^{2}$ Nombre total de déclarations d'effets secondaires suivant l'immunisation $=528$

(deux déclarations d'effets secondaires suivant l'immunisation exclues en raison d'un âge inconnu)

${ }^{3}$ Utilisation des projections de population de 2014-2015 ( $\mathrm{N}=13672718$ )

${ }^{4}$ Le taux de déclaration est calculé au moyen des dénominateurs suivants : estimations de la population de 2012-2013 en 2012 et de 2013-2014 en 2013, et projections de 2014 pour 2014-2015

La plupart des effets indésirables ont été signalés après I'administration du vaccin antigrippal seulement $(93,6 \% ; n=494)$ et 34 déclarations concernaient l'administration concomitante avec d'autres vaccins, le plus souvent le vaccin polysaccharidique 23-valent contre le pneumocoque (Pneu-P-23) ( $n=15)$ et le vaccin contre la diphtérie, la coqueluche acellulaire et le tétanos (dcaT) $(n=7)$. Les effets les plus souvent déclarés étaient des réactions au site d'injection, qui ont été documentés dans 36,2\% de toutes les déclarations. D'autres types d'effets fréquemment déclarés comprenaient les réactions allergiques cutanées $(21,1 \%)$ et les éruptions cutanées (17,3\%) (tableau 2). Les taux de déclaration propres à un effet les plus élevés étaient semblables au volume de déclarations, avec les taux les plus élevés concernant une douleur, une rougeur et un gonflement au site d'injection, des réactions allergiques cutanées et des éruptions cutanées $(1,3,0,9$ et 0,8 pour 100000 doses distribuées, respectivement). Les événements médicaux importants $(n=27)$ comprenaient l'anaphylaxie $(n=22)$, la thrombocytopénie $(n=1)$ et la rhabdomyolyse aiguë $(n=1)$, ainsi que trois effets graves décrits ci-dessous. Les déclarations d'effets pris en charge comme anaphylaxie concernaient des cas dont l'âge se situait entre 4 et 84 ans, concernaient principalement des femmes $(81,8 \% ; n=18)$ et aucune n'a été 
considérée comme grave. Huit cas $(36,4 \%)$ répondaient à la définition de cas d'anaphylaxie de Brighton Collaboration (un niveau I, six niveau II, un niveau III) et les autres 14 déclarations $(63,6 \%)$ ne contenaient pas suffisamment de données probantes pour répondre à la définition. Fondé sur les effets qui

Tableau 2 : Nombre, distribution en pourcentage et taux de déclaration des effets secondaires suivant l'immunisation contre la grippe en Ontario, par catégorie d'effet secondaire, de 2012-2013 à 2014-2015

\begin{tabular}{|c|c|c|c|c|c|}
\hline $\begin{array}{c}\text { Type } \\
\text { d'effets } \\
\text { secondaires }{ }^{1}\end{array}$ & $\begin{array}{c}\text { Effets } \\
\text { secondaires }^{2}\end{array}$ & $\begin{array}{c}\text { Nombre de } \\
\text { déclarations } \\
\text { d'effets } \\
\text { secondaires } \\
\text { suivant } \\
\text { I'immunisation }\end{array}$ & $\begin{array}{c}\text { Taux de } \\
\text { déclaration }\end{array}$ & \begin{tabular}{|c|} 
Pourcentage \\
de toutes \\
les \\
déclarations \\
d'effets \\
secondaires \\
suivant \\
I'immunisation
\end{tabular} & $\begin{array}{c}\text { Nombre de } \\
\text { déclarations } \\
\text { graves }\end{array}$ \\
\hline \multirow[t]{9}{*}{$\begin{array}{l}\text { Réaction au site } \\
\text { d'injection }\end{array}$} & Total & 190 & 1,6 & 36,2 & 8 \\
\hline & Cellulite & 30 & 0,2 & 5,7 & 6 \\
\hline & Abcès infecté & 2 & $<0,1$ & 0,4 & 1 \\
\hline & Nodule & 4 & $<0,1$ & 0,8 & 0 \\
\hline & $\begin{array}{l}\text { Douleur/rougeur/ } \\
\text { gonflement au site } \\
\text { d'injection' }^{1}\end{array}$ & 162 & 1,3 & 30,9 & 3 \\
\hline & $\begin{array}{r}\text { Douleur/rougeur/ } \\
\text { gonflement } \\
\text { s'étendant au-delà } \\
\text { de l'articulation la } \\
\text { plus proche }\end{array}$ & 40 & 0,3 & 7,6 & 1 \\
\hline & $\begin{array}{r}\text { Douleur/rougeur/ } \\
\text { gonflement de } \\
\text { moins de quatre } \\
\text { jours } \\
\end{array}$ & 7 & 0,1 & 1,3 & 0 \\
\hline & $\begin{array}{r}\text { Douleur/rougeur/ } \\
\text { gonflement de } \\
\text { quatre jours ou plus }\end{array}$ & 129 & 1,1 & 24,6 & 2 \\
\hline & Abcès stérile & 2 & $<0,1$ & 0,4 & 0 \\
\hline \multirow[t]{11}{*}{$\begin{array}{l}\text { Réactions } \\
\text { générales }\end{array}$} & Total & 156 & 1,3 & 29,7 & 7 \\
\hline & $\begin{array}{l}\text { Adénopathie/ } \\
\text { lymphadénopathie }\end{array}$ & 10 & 0,1 & 1,9 & 0 \\
\hline & Arthrite/arthralgie & 16 & 0,1 & 3 & 1 \\
\hline & $\begin{array}{l}\text { Fièvre de } 38^{\circ} \mathrm{C} \\
\text { conjointement } \\
\text { avec un autre } \\
\text { effet à déclaration } \\
\text { obligatoire }\end{array}$ & 42 & 0,3 & 8 & 6 \\
\hline & $\begin{array}{l}\text { Épisode } \\
\text { hypotonique- } \\
\text { hyporéactif (EHH) }\end{array}$ & 1 & $<0,1$ & 0,2 & 0 \\
\hline & Parotidite & 1 & $<0,1$ & 0,2 & 0 \\
\hline & $\begin{array}{l}\text { Cris/pleurs } \\
\text { persistants }\end{array}$ & 1 & $<0,1$ & 0,2 & 0 \\
\hline & Éruption cutanée & 91 & 0,8 & 17,3 & 1 \\
\hline & $\begin{array}{l}\text { Vomissements/ } \\
\text { diarrhée graves }^{6}\end{array}$ & 14 & 0,1 & 2,7 & 1 \\
\hline & $\begin{array}{l}\text { Syncope avec } \\
\text { blessure }^{6}\end{array}$ & 1 & $<0,1$ & 0,2 & 0 \\
\hline & Thrombocytopénie & 1 & $<0,1$ & 0,2 & 0 \\
\hline \multirow[t]{5}{*}{ Allergies } & Total & 145 & 1,2 & 27,6 & 1 \\
\hline & $\begin{array}{l}\text { Réaction allergique } \\
\text { - peau }\end{array}$ & 111 & 0,9 & 21,1 & 1 \\
\hline & $\begin{array}{l}\text { Réaction allergique } \\
\text { - autres }\end{array}$ & 6 & $<0,1$ & 1,1 & 0 \\
\hline & $\begin{array}{l}\text { Effet pris en } \\
\text { charge comme } \\
\text { anaphylaxie }^{6}\end{array}$ & 22 & 0,2 & 4,2 & 0 \\
\hline & $\begin{array}{l}\text { Syndrome oculo- } \\
\text { respiratoire }\end{array}$ & 11 & 0,1 & 2,1 & 0 \\
\hline \multirow[t]{7}{*}{$\begin{array}{l}\text { Événements } \\
\text { neurologiques }\end{array}$} & Total & 32 & 0,3 & 6,1 & 8 \\
\hline & $\begin{array}{l}\text { Anesthésie/ } \\
\text { paresthésie }^{6}\end{array}$ & 10 & 0,1 & 1,9 & 2 \\
\hline & Paralysie de Bell & 4 & $<0,1$ & 0,8 & 0 \\
\hline & Convulsions/crise & 9 & 0,1 & 1,7 & 3 \\
\hline & $\begin{array}{l}\text { Encéphalopathie/ } \\
\text { encéphalite }\end{array}$ & 2 & $<0,1$ & 0,4 & 1 \\
\hline & $\begin{array}{l}\text { Syndrome de } \\
\text { Guillain-Barré } \\
\text { (SGB) }\end{array}$ & 3 & $<0,1$ & 0,6 & 2 \\
\hline & $\begin{array}{l}\text { Paralysie autre que } \\
\text { la paralysie de Bell }\end{array}$ & 4 & $<0,1$ & 0,8 & 0 \\
\hline $\begin{array}{l}\text { Autres } \\
\text { événements } \\
\text { graves/ } \\
\text { inhabituels }\end{array}$ & Total & 105 & 0,9 & 20,0 & 8 \\
\hline
\end{tabular}

Les notes du tableau

Les catégories d'effets secondaires regroupent des types précis d'effets secondaires et ne son pas mutuellement exclusives. Les déclarations faisant état de plus d'un effet secondaire d'une catégorie ne sont comptées qu'une seule fois dans le total de cette catégorie. Par conséquent le total à l'intérieur d'une catégorie ne correspond pas forcément à la somme des différents cas d'effets secondaires de cette catégorie

${ }^{2} \mathrm{~N}$ 'inclut que les effets secondaires qui ont eu lieu au moins une fois

${ }^{3}$ Chaque déclaration d'effets secondaires suivant l'immunisation peut faire part de plusieurs effets secondaires qui ne sont pas mutuellement exclusifs. La somme des pourcentages n'est pas égale à $100 \%$. Le dénominateur est le nombre total de déclarations d'effets secondaires suivant l'immunisation confirmé entre les saisons grippales de 2012-2013 et de 2014-2015.

Trois déclarations n'étaient associées à aucun effet secondaire, et ont donc été exclues du nombre total de déclarations d'effets secondaires suivant l'immunisation confirmé dans la présente analyse $(n=525)$

${ }^{4}$ Les taux de déclaration sont calculés au moyen du nombre total de doses distribuées entre les saisons grippales de 2012-2013 et de 2014-2015

Inclut les valeurs de "douleur/rougeur/gonflement de moins de quatre jours ", de "réaction allergique - cardiovasculaire » et de "réaction allergique - respiratoire ", qui ont cessé d'être prises en compte dans le SISP-i le $1^{\text {er }}$ janvier 2013

${ }^{6}$ Ces effets ont été ajoutés comme nouvelles valeurs au champ des réactions secondaires dans le SISP-i le $1^{\text {er }}$ janvier 2013

répondaient à la définition de Brighton, le taux de déclaration d'anaphylaxie était de 0,7 cas pour un million de doses distribuées.

Il y a eu 20 déclarations d'effets secondaires suivant l'immunisation graves dans toutes les saisons, ce qui représente $3,8 \%$ de tous les effets secondaires suivant l'immunisation ainsi qu'un taux de déclaration de 1,6 cas pour un million de doses distribuées. La proportion de déclarations qui ont été classées comme graves par saison a diminué de façon constante $(5,0 \%$, $3,6 \%$ et $2,3 \%$ de 2012-2013 à 2014-2015, respectivement), de même que les taux de déclaration grave (de 2,5 cas pour un million de doses distribuées en 2012-2013 à 1,8 cas et 0,7 cas pour un million de doses distribuées en 2013-2014 et 2014-2015, respectivement). Les déclarations graves concernaient des personnes de 1 à 81 ans, et le plus grand nombre de rapports concernaient des adultes âgés de 50 à 64 ans et de 65 à 79 ans, ainsi que des enfants de 1 à 4 ans (quatre déclarations pour chaque groupe d'âge). Les femmes représentaient $60 \%$ des déclarations graves. Le type d'effet le plus fréquent parmi les effets secondaires suivant l'immunisation graves était la cellulite (nécessitant une hospitalisation pour un traitement antibiotique par voie intraveineuse) $(n=6)$. Voici $d$ 'autres effets graves: convulsions ( $n=2$; une convulsion fébrile chez un enfant et une convulsion afébrile chez un adulte), anesthésie/paresthésie ( $n=2)$, syndrome de Guillain-Barré $(n=3)$, panuvéite bilatérale ( $n=2$; I'un des cas avait aussi un diagnostic de syndrome de Guillain-Barré), encéphalite $(n=1)$, polyneuropathie inflammatoire démyélinisante chronique $(n=1)$, pseudo-polyarthrite rhyzomélique $(n=1)$, éruption cutanée fébrile $(n=1)$, abcès infecté $(n=1)$ et une déclaration de l'apparition soudaine de limites de mobilité avec une résolution spontanée. Aucun décès n'a été signalé.

La plupart des effets secondaires suivant l'immunisation ont été signalés par des professionnels de la santé $(63,8 \%: 25,3 \%$ par des médecins et 38,5\% par d'autres professionnels de la santé), puis par des autodéclarations et des déclarations par des membres de la famille $(12,1 \%$ et $8,2 \%$, respectivement). Pour les déclarations dont les renseignements sur l'utilisation des soins de santé étaient indiqués, $68,2 \%(n=353)$ des cas ont cherché une consultation médicale externe, $23,1 \%(n=121)$ ont été observés au service des urgences et 3,9\% $(n=20)$ ont été hospitalisés. Dans la plupart des cas, la personne était rétablie au moment de la déclaration (70,0 \%, $n=336), 26,0 \%$ n'étaient pas encore rétablis (mais un rétablissement complet était prévu) et il a été documenté que $4,0 \%(n=19)$ avaient des effets 
résiduels. Parmi toutes les déclarations, il a été indiqué que deux personnes étaient enceintes; ces deux cas avaient des effets bénins qui se sont entièrement dissipés. Il y a eu six déclarations d'effets secondaires suivant l'immunisation où une erreur d'immunisation a été notée. Ces six cas concernaient tous une mauvaise technique d'administration du vaccin (p. ex. injection intramusculaire administrée trop haut, aiguille trop courte) causant une douleur prolongée au site d'injection et une déclaration grave de cellulite.

\section{Discussion}

Cette évaluation des effets secondaires suivant l'immunisation déclarés en Ontario durant les trois dernières saisons grippales correspond au profil d'innocuité des vaccins antigrippaux bien établi. Le taux de déclaration des effets secondaires suivant l'immunisation contre la grippe en Ontario de 4,4 cas pour 100000 doses distribuées entre les saisons grippales de 2012-2013 et de 2014-2015 représent l'un des taux de déclaration propres à un vaccin le plus faible de tous les vaccins financés par l'État de la province (19). Ce taux était également plus faible que le taux national le plus récent de déclaration d'effets secondaires suivant l'immunisation contre la grippe (8,9 cas pour 100000 doses distribuées; de 2012-2013 à 2014-2015) (20), ce qui laisse supposer une sous-déclaration en Ontario comparativement aux autres provinces et territoires canadiens.

La diminution des taux de déclaration observée en 2014-2015 par rapport aux années précédentes a été inattendue et n'est pas entièrement comprise. Elle correspondait toutefois à une diminution observée à l'échelle nationale au cours de la même saison. Une partie de la diminution pourrait avoir été causée par les changements aux définitions de cas d'effets secondaires suivant l'immunisation, qui ont été apportés au cours de la première saison en 2013 (p. ex. limiter la déclaration des effets comprenant de la douleur, une rougeur ou un gonflement au point d'injection à ceux qui durent quatre jours ou plus). Les retards dans les déclarations peuvent jouer un rôle, bien qu'en Ontario, la proportion de déclarations tardives (p. ex. déclarations après le 31 août) pour 2012-2013 et 2013-2014 était minime $(3,5 \%$ et $1,5 \%$, respectivement), et une proportion semblable de déclarations tardives pour la saison 2014-2015 causerait quand même un taux de déclaration d'effets secondaires suivant l'immunisation plus faible.

Les taux de déclaration des effets secondaires suivant l'immunisation contre la grippe en fonction de la population pour un groupe d'âge précis étaient généralement ceux prévus, avec des taux et des proportions plus élevés d'effets secondaires suivant l'immunisation (comparativement à la répartition de la population) dans les groupes d'âge les plus jeunes. La prédominance observée chez les femmes du nombre de déclarations d'effets secondaires suivant l'immunisation avait déjà été observée en Ontario $(21,22)$ et dans d'autres systèmes de surveillance passive des effets secondaires suivant I'immunisation (23-25), même si elle semble particulièrement frappante dans l'évaluation actuelle $(93,3 \%$ et $86,5 \%$ chez les 18 à 49 ans et les 50 à 64 ans, respectivement). Les raisons de ce phénomène sont probablement multifactorielles. Les différences entre les taux de vaccination des hommes et des femmes peuvent jouer un rôle, surtout en raison du fait que les travailleurs de la santé (un groupe cible pour le vaccin antigrippal) sont plus susceptibles d'être des femmes. Cependant, les estimations provinciales de l'Enquête sur la santé dans les collectivités canadiennes (12 ans et plus; immunisation contre la grippe il y a moins d'un an) laissent entendre qu'il y a seulement une légère prédominance des femmes parmi les personnes immunisées $(54,1 \%$ des cas étaient des femmes) (26). D'autres facteurs possibles comprennent les différences entre les comportements des hommes et des femmes pour obtenir des soins de santé (27-29) une fois qu'un effet secondaire suivant l'immunisation survient, ainsi qu'une réaction biologique différente aux vaccins $(30,31)$. La différence entre les déclarations d'effets secondaires suivant l'immunisation selon le sexe était moins prononcée pour les effets secondaires suivant l'immunisation graves, dont $60 \%$ concernaient des femmes.

Les réactions au site d'injection étaient le type d'effet le plus fréquemment déclaré dans cette évaluation, ce qui est conforme aux essais cliniques et à la surveillance après commercialisation des produits de vaccination antigrippale administrés par injection intramusculaire $(4,5,23,32)$. Contrairement au volume relativement élevé de réactions au site d'injection parmi tous les effets secondaires suivant l'immunisation, le taux de déclaration était plutôt faible (1,6 cas pour 100000 doses distribuées). Bien que ce taux sous-estime probablement le nombre réel d'occurrences des réactions localisées, une certaine mesure de sous-déclaration est attendue pour ces effets, puisqu'ils sont généralement bénins et se résorbent d'eux-mêmes. Les réactions allergiques étaient également fréquemment déclarées, dont la plupart étaient des réactions allergiques cutanées, ce qui est conforme aux évaluations précédentes des effets secondaires suivant l'immunisation $(19,20,23)$. Un petit nombre de réactions allergiques ont été classées comme effet pris en charge comme anaphylaxie. D'après les déclarations répondant à la définition de Brighton, le taux de déclaration de l'anaphylaxie était comparable, quoique légèrement inférieur, au taux prévu d'anaphylaxie après des vaccinations antigrippales, qui a été estimé à environ un cas pour un million de doses de vaccin $(33,34)$.

Comme on pouvait s'y attendre, les effets graves suivant l'administration d'un vaccin antigrippal durant cette période étaient rares et les plus souvent liés à des effets connus comme étant rarement signalés après l'administration de vaccins antigrippaux. Par exemple, le syndrome de Guillain-Barré est un effet rare qui est systématiquement déclaré dans le cadre de la surveillance après commercialisation des vaccins antigrippaux, y compris en Ontario, où il y a eu trois déclarations de syndrome de Guillain-Barré suivant l'administration d'un vaccin antigrippal en trois ans. Bien que les données probantes sur la vaccination antigrippale et le syndrome de Guillain-Barré ne soient pas suffisantes pour confirmer ou infirmer l'existence d'un lien de causalité (35), le risque absolu d'environ un cas en excès pour un million de vaccins (36-38) est beaucoup plus faible que celui associé à la grippe $(39,40)$. Il convient de noter qu'au cours de cette période, on a déclaré deux cas de panuvéite bilatérale qui ont été décrits plus en détail par Manusow et ses collègues (41). La panuvéite est une pathologie rare le plus souvent liée à des causes infectieuses ou inflammatoires (42), bien qu'il n'existe aucun lien de causalité avec les vaccins (43).

Les limites de l'analyse comprennent celles qui sont inhérentes à de nombreux systèmes de surveillance passive des effets 
secondaires suivant l'immunisation, telles que la qualité des données, I'exhaustivité et le biais de déclaration $(23,24)$. II a déjà été démontré que la sous-déclaration des effets secondaires suivant l'immunisation est plus fréquente en Ontario que dans les autres provinces et territoires (19), et cela est encore une fois proposé ici. Les taux de déclaration ont été calculés en se servant comme dénominateur de la population totale ou du nombre total de doses distribuées, tous deux substituts des doses administrées en l'absence d'un registre provincial $d^{\prime}$ immunisation en fonction de la population. Bien que les doses distribuées soient largement utilisées dans les analyses des systèmes de surveillance passive des effets secondaires suivant I'immunisation $(23,24)$, il est possible de sous-estimer les taux de déclaration des effets secondaires suivant l'immunisation si la perte de vaccins n'est pas bien documentée ou comprise. Puisque l'analyse des tendances comprend seulement trois saisons, l'interprétation peut être limitée. Les analyses des saisons supplémentaires sur une plus longue période permettront d'éclairer davantage l'évaluation continue des tendances en matière de déclaration des effets secondaires suivant l'immunisation.

\section{Conclusion}

La présente évaluation a révélé que les vaccins antigrippaux administrés dans le cadre du Programme universel de vaccination contre la grippe de l'Ontario ont causé un faible taux d'effets secondaires déclarés. La plupart des effets signalés étaient légers et se sont complètement dissipés. Aucun problème imprévu lié à l'innocuité n'a été cerné. Ces résultats sont conformes au très bon profil d'innocuité des vaccins antigrippaux utilisés au Canada et à l'échelle internationale. La surveillance continue est importante pour surveiller les signes de problème lié à l'innocuité et les tendances de déclaration au fil du temps, particulièrement avec l'introduction des nouveaux vaccins antigrippaux, et pour maintenir la confiance du public et des professionnels quant à l'innocuité des vaccins antigrippaux. Une analyse plus approfondie est nécessaire pour comprendre la diminution du taux au fil du temps et la sous-déclaration dans le système de surveillance afin d'optimiser les données de surveillance des effets secondaires suivant l'immunisation en Ontario.

\section{Remerciements}

Nous tenons à remercier sincèrement les membres du personnel des bureaux de santé publique dans l'ensemble de la province pour les efforts qu'ils ont réalisés pour enquêter sur les effets secondaires suivant l'immunisation et pour les déclarer. Ces efforts sont essentiels pour l'évaluation du Programme universel de vaccination contre la grippe et de l'innocuité des vaccins antigrippaux en Ontario.

\section{Conflit d'intérêts}

Aucun.

\section{Financement}

Ce projet a été financé par Santé publique Ontario.

\section{Références}

1. Comité consultatif national de l'immunisation (CCNI). Déclaration sur la vaccination antigrippale pour la saison 20152016. Ottawa ON: PHAC; 2015 [Date de modification: le 8 mars 2016]. http://www.phac-aspc.gc.ca/naci-ccni/flu-2015grippe-fra.php.

2. Santé Canada. Médicaments et produits de santé. Ottawa ON: Santé Canada; 2016 [Date de modification : le 17 juil 2015].

3. Ontario Ministry of Health and Long-Term Care (OMHLTC). Health care provider Q \& A: 2015/2016 Universal Influenza Immunization Program. Toronto ON: OMHLTC; 2015.

4. National Advisory Committee on Immunization (NACl). An Advisory Committee Statement (ACS). Influenza vaccine effectiveness, immunogenicity, and safety in healthy adults 1964 years old. Ottawa ON: PHAC; 2014 [updated 2013 Apr 3].

5. World Health Organization (WHO). Information sheet. Observed rate of vaccine reactions. Influenza vaccine. Geneva: WHO; 2012. http://www.who.int/vaccine_safety/initiative/tools/ Influenza_Vaccine_rates_information_sheet.pdf.

6. Dube E, Gagnon D, Zhou Z, Deceuninck G. Parental vaccine hesitancy in Quebec (Canada). PLoS Curr 2016;Mar 7;8.

7. Government of Canada. Vaccine coverage in Canadian children: Highlights from the 2013 Childhood National Immunization Coverage Survey (cNICS). Ottawa ON: Government of Canada; 2014 [Date de modification : le 26 juil 2016].

8. Nowak GJ, Sheedy K, Bursey K, Smith TM, Basket M. Promoting influenza vaccination: Insights from a qualitative meta-analysis of 14 years of influenza-related communications research by U.S. Centers for Disease Control and Prevention (CDC). Vaccine 2015:Jun 4;33(24):2741-2756.

9. Prematunge C, Corace K, McCarthy A, Nair RC, Roth V, Suh $K N$, et al. Qualitative motivators and barriers to pandemic vs. seasonal influenza vaccination among healthcare workers: A content analysis. Vaccine 2014 Dec 12;32(52):7128-7134.

10. Naleway AL, Henkle EM, Ball S, Bozeman S, Gaglani MJ, Kennedy ED, et al. Barriers and facilitators to influenza vaccination and vaccine coverage in a cohort of health care personnel. Am J Infect Control 2014 Apr;42(4):371-375.

11. Quach S, Pereira JA, Kwong JC, Quan S, Crowe L, Guay M, et al. Immunizing health care workers against influenza: $A$ glimpse into the challenges with voluntary programs and considerations for mandatory policies. Am J Infect Control 2013 Nov;41(11):1017-1023.

12. Ontario Ministry of Health and Long-Term Care. Infectious diseases protocol. Appendix B: Provincial case definitions for reportable diseases. Disease: Adverse events following immunization. Toronto ON: OMHLTC; 2015.

13. International Conference on Harmonisation (ICH). ICH Harmonised tripartite international guideline: Clinical safety data management: Definitions and standards for expedited reporting. E2A. Geneva: ICH; 1994. http://www.fda.gov.ph/ attachments/article/99526/E2A\%20Step4.pdf. 
14. International Conference on Harmonisation (ICH). ICH harmonised tripartite guideline: Post-approval safety data management: Definitions and standards for expedited reporting. E2D. Geneva: ICH; 2003. https://www.ich.org/ fileadmin/Public_Web_Site/ICH_Products/Guidelines/Efficacy/ E2D/Step4/E2D_Guideline.pdf.

15. Ruggeberg JU, Gold MS, Bayas JM, Blum MD, Bonhoeffer J, Friedlander S, et al. Anaphylaxis: Case definition and guidelines for data collection, analysis, and presentation of immunization safety data. Vaccine 2007 Aug;25(31):5675-5684.

16. Government of Ontario. Health Protection and Promotion Act. R.S.O. 1990, Chapter H.7. (2014).

17. Ontario Ministry of Health and Long-Term Care. Population estimates. intelliHEALTH Ontario. Ottawa ON: Statistics Canada; 2015.

18. Ontario Ministry of Health and Long-Term Care. Population projections. intelliHEALTH, Ontario. Ottawa ON: Ministry of Finance; 2015.

19. Public Health Ontario (PHO). Annual report on vaccine safety in Ontario, 2014. Toronto ON: PHO; 2015.

20. Gendron MP, Ahmadipour N, Nkanza J, Pless R, Bancej C. Surveillance of adverse events following immunization with influenza vaccines in Canada, 2012-2015. Annual Conference on Vaccine Research. Baltimore MD. April 18-20, 2016.

21. Public Health Ontario (PHO). Annual report on vaccine safety in Ontario, 2012. Toronto ON: PHO; 2014.

22. Harris T, Wong K, Fediurek J, Deeks S. Assessment of sexspecific differences in adverse events following immunization reporting in Ontario, Canada. International Conference on Pharmacoepidemiology and Therapeutic Risk Management. Boston, MA. Aug.22-26, 2015.

23. Law BJ, Laflèche J, Ahmadipour N, Anyoti H. Rapport annuel du Système canadien de surveillance des effets secondaires suivant d'immunisation (SCSESSI) pour les vaccins administrés en 2012. Relevé des maladies transmissibles au Canada 2014;40-Supp-3:S7-23. http://www.phac-aspc.gc.ca/publicat/ ccdr-rmtc/14vol40/dr-rm40s-3/assets/pdf/14vol40s-3-fra.pdf.

24. Zhou W, Pool V, Iskander JK, English-Bullard R, Ball R, Wise $R P$, et al. Surveillance for safety after immunization: Vaccine Adverse Event Reporting System (VAERS): United States, 19912001. MMWR Surveill Summ 2003 Jan 24;52(1):1-24.

25. Lawrence G, Boyd I, Mclntyre P, Isaacs D. Surveillance of adverse events following immunisation: Australia 2002 to 2003. Commun Dis Intell Q Rep 2004;28(3):324-338.

26. Statistique Canada. Tableau 105-0501. Profil d'indicateurs de la santé, estimations annuelles, selon le group d'âge et le sexe, Canada, provinces, territoires, régions sociosanitaires (limites de 2013) et groupes de régions homologues. CANSIM. Ottawa ON: Statistique Canada; 2016. [Date de modification : le 22 avr 2016]. http://www5.statcan.gc.ca/cansim/ a26?lang=fra\&id=1050501.

27. Bertakis KD, Azari R, Helms LJ, Callahan EJ, Robbins JA. Gender differences in the utilization of health care services. J Fam Pract 2000 Feb;49(2):147-152.

28. Galdas PM, Cheater F, Marshall P. Men and health help-seeking behaviour: Literature review. J Adv Nurs 2005 Mar;49(6):616623.
29. Smith J, Braunack-Mayer A, Wittert G. What do we know about men's help-seeking and health service use? Med J Aust 2006;184(2):81-83.

30. Klein $S L$, Jedlicka $A$, Pekosz $A$. The $X$ s and $Y$ of immune responses to viral vaccines. Lancet Infect Dis 2010 May;10(5):338-349.

31. Klein SL, Poland GA. Personalized vaccinology: One size and dose might not fit both sexes. Vaccine 2013 May 28;31(23):2599-2600.

32. Centers for Disease Control and Prevention (CDC). Prevention and control of seasonal influenza with vaccines. Recommendations of the Advisory Committee on Immunization Practices-United States, 2013-2014. MMWR Recomm Rep 2013 Sep 20;62(RR-07):1-43.

33. McNeil MM, Weintraub ES, Duffy J, Sukumaran L, Jacobsen SJ, Klein NP, et al. Risk of anaphylaxis after vaccination in children and adults. J Allergy Clin Immunol 2015 Sep 28.

34. Bohlke K, Davis RL, Marcy SM, Braun MM, DeStefano F, Black $\mathrm{SB}$, et al. Risk of anaphylaxis after vaccination of children and adolescents. Pediatrics 2003 Oct;112(4):815-820.

35. Stratton K, Ford A, Rusch E, Wright Clayton E. National Academy of Sciences. Adverse effects of vaccines: Evidence and causality. Washington DC: NAM; 2011. http://www.nap. edu/catalog/13164/adverse-effects-of-vaccines-evidence-andcausality.

36. Haber P, DeStefano F, Angulo F, et al. Guillain-Barré syndrome following influenza vaccination. JAMA 2004 Nov;292:2478-81.

37. Juurlink D, Stukel T, Kwong J, et al. Guillain-Barré syndrome after influenza vaccination in adults: A population-based study. Arch Intern Med 2006;166:2217-21.

38. Prothro C, Kudish K, Fielin M. Preliminary results: Surveillance for Guillain-Barré syndrome after receipt of influenza A (H1N1) 2009 monovalent vaccine - United States, 2009-2010. MMWR. 2010;59:657-61.

39. Kwong J, Vasa P, Campitelli M, et al. Risk of Guillain-Barré syndrome after seasonal influenza vaccination and influenza health-care encounters: A self-controlled study. Lancet Infect Dis 2013;13(9):769-76.

40. Hawken S, Kwong JC, Deeks SL, Crowcroft NS, McGeer AJ, Ducharme R, et al. Simulation study of the effect of influenza and influenza vaccination on risk of acquiring Guillain-Barré syndrome. Emerg Infect Dis 2015 Feb;21(2):224-231.

41. Manusow JS, Rai A, Yeh S, Mandelcorn ED. Two cases of panuveitis with orbital inflammatory syndrome after influenza vaccination. Can J Ophthalmol 2015 Oct;50(5):e71-4.

42. Rosenbaum J. Uveitis: Etiology, clinical manifestations, and diagnosis. Alphen aan den Rijn NL: Wolters Kluwer; 2015. http://www.uptodate.com/contents/uveitis-etiology-clinicalmanifestations-and-diagnosis?source=search_result\&search=uv eitis\&selectedTitle=1 150.

43. National Academies of Sciences, Engineering and Medicine. Adverse effects of vaccines: Evidence and causality. Washington DC: NAM; 2011. http://www.nationalacademies. org/hmd/Reports/2011/Adverse-Effects-of-Vaccines-Evidenceand-Causality.aspx. 